\title{
Clinically Important Improvement in Function Is Common in People with or at High Risk of Knee OA: The MOST Study
}

\author{
DANIEL K. WHITE, JULIE J. KEYSOR, MICHAEL P. LAVALLEY, CORA E. LEWIS, JAMES C. TORNER, \\ MICHAEL C. NEVITT, and DAVID T. FELSON
}

\begin{abstract}
Objective. To calculate the frequency of clinically important improvement in function over 30 months and identify risk factors in people who have or are at risk of knee osteoarthritis (OA). Methods. Subjects were from the Multicenter Osteoarthritis (MOST), a longitudinal study of persons with or at high risk of knee OA. We defined minimal clinically important improvement (MCII) with the Western Ontario McMaster Universities Osteoarthritis Index (WOMAC) physical function using 3 different methods. Baseline risk factors tested for improvement included age, sex, educational attainment, presence of radiographic knee OA (ROA), the number of comorbidities, body mass index (BMI), knee pain, walking speed, isokinetic knee extensor strength, depressive symptoms, physical activity, and medication usage. We used logistic regression to evaluate the association of baseline risk factors with MCII.

Results. Of the 1801 subjects (mean age 63 yrs, BMI = 31, 63\% women), most had mild limitations in baseline function (WOMAC $=19 \pm 11$ ). Regardless of how defined, a substantial percentage of subjects $(24 \%-39 \%)$ reached MCII at 30 months. Compared to their counterparts, people with MCII were less likely to have ROA and to use medications, and were more likely to have a lower BMI, less knee pain, a faster walking speed, more knee strength, and fewer depressive symptoms. After adjustment, MCII was $40 \%$ to $50 \%$ less likely in those with ROA, and 1.9 to 2.0 times more likely in those walking 1.0 meters/second faster than counterparts.

Conclusion. Clinically important improvement is frequent in people with or at high risk of knee OA. The absence of ROA and a faster walking speed appear to be associated with clinically important improvements. (First Release April 15 2010; J Rheumatol 2010;37:1244-51; doi:10.3899/ jrheum.090989)
\end{abstract}

\section{Key Indexing Terms:} OSTEOARTHRITIS

\section{FUNCTIONAL LIMITATION}

\section{REHABILITATION}

The natural history of knee pain and osteoarthritis (OA) often leads to difficulty performing functional activities ${ }^{1}$, yet a number of persons maintain a high level of functioning $^{2}$, improve ${ }^{3,4}$, or recover from previous limitations ${ }^{5}$.

From the Boston University (BU) School of Medicine, Clinical Epidemiology Research and Training Unit; BU Sargent College of Health and Rehabilitation Sciences, Physical Therapy and Athletic Training; $B U$ School of Public Health, Boston, Massachusetts; University of Alabama at Birmingham, Birmingham, Alabama; Department of Epidemiology, University of Iowa, Iowa City, Iowa; and University of California San Francisco, San Francisco, California, USA.

Supported by NIH U01 AG18820, U01 AG 18832, U01 AG 18947, U01 AG 19069, NIDRR ARRT Grant H133P050001, AR47785, AR007598, and the ARHP New Investigator Award.

D.K. White, PT, ScD; D.T. Felson, MD, MPH, BU School of Medicine, Clinical Epidemiology Research and Training Unit; J.J. Keysor, PT, PhD, $B U$ Sargent College of Health and Rehabilitation Sciences, Physical Therapy and Athletic Training; M.P. LaValley, PhD, BU School of Public Health; C.E. Lewis, MD, University of Alabama at Birmingham; J.C. Torner, PhD, Department of Epidemiology, University of Iowa; M.C. Nevitt, PhD, University of California San Francisco.

Address correspondence to D. White, Clinical Epidemiology Research and Training Unit, Boston University School of Medicine, 650 Albany Street $X$ 200, Boston, MA 02118,USA.E-mail: white.daniel@gmail.com

Accepted for publication January 21, 2010.
While this may seem contrary to the chronic progressive nature of $\mathrm{OA}$, large observational studies from the past decade confirm this ${ }^{2,6,7}$. Closer examination of these studies reveals, however, that the magnitude or clinical significance of improvement is largely unknown. In particular, it is not clear how much of the improvement in these and other studies represents change that is relevant to the patient or healthcare provider.

Beaton and others have emphasized the need to differentiate between improvements in outcome that are merely statistically significant versus those that are clinically meaningful or important ${ }^{8}$. One method to qualify the clinical significance of improvement is to use a minimal clinically important improvement (MCII) threshold, which represents the smallest improvement that is important from the subject's perspective ${ }^{9}$. Although different thresholds of MCII for the Western Ontario McMaster Universities Osteoarthritis Index (WOMAC) physical function scale have been reported ${ }^{9,10}$, to date only one study with 44 subjects has examined the longitudinal occurrence of MCII in function in people with symptomatic hip or knee OA. That study found that 8 subjects had meaningful improvement as 
measured by the WOMAC physical function scale over 5 years ${ }^{11}$. We studied the frequency of clinically important improvement within a larger cohort of people with knee OA.

There is much literature examining risk factors of functional decline for persons with knee OA, but little is known about factors related to improvement. Research has identified these factors with decline in function in people with knee pain, symptomatic knee OA, and older individuals: age $^{4,12}$, body mass index $(\mathrm{BMI})^{12-15}$, pain at base$\operatorname{line}^{4,12-14,16}$, and walking speeds ${ }^{17,18}$. It is not clear whether these factors for decline are also associated with clinically important improvements in function. In particular, we are interested in identifying baseline risk factors associated with MCII to help clinicians better identify which of their patients evaluated for the first time are likely to make future improvements in function. Additionally, recognizing modifiable baseline risk factors associated with improvement may provide therapeutic targets for interventions to bring about functional gains. We will examine whether factors of decline are also important for meaningful improvement in function.

Our purpose was to examine the frequency of clinically important improvement in WOMAC physical function over 30 months using 3 definitions of MCII in people with or at risk of symptomatic knee OA with existing limitations in function, and to examine baseline factors associated with clinically meaningful improvement.

\section{MATERIALS AND METHODS}

Participants were recruited from the Multicenter Osteoarthritis (MOST) study, a large multicenter prospective cohort study of 3026 communitydwelling persons who were at high risk of developing symptomatic knee $\mathrm{OA}$ at baseline. MOST was designed to evaluate the effects of a variety of potential risk factors on the occurrence and progression of radiographic and symptomatic knee OA. Subjects aged 50 to 79 years were recruited from Birmingham, Alabama, and Iowa City, Iowa, USA. Baseline assessments took place between May 2003 and March 2005, and followup assessments 30 months later. Participants were defined as being at risk of developing knee OA based on known risk factors, including older age, female sex, previous knee injury or operation, and high body weight. A more detailed description of recruitment and sampling for MOST has been published ${ }^{19}$.

We focused on MOST subjects with at least a minimal degree of self-reported functional limitation at baseline to permit us to study possible improvement in these limitations. We defined this as a baseline WOMAC physical function score of at least $4 / 68$, which is consistent with a previous definition of minimal limitation in function ${ }^{2}$. We anticipated that subjects undergoing a knee or hip replacement would likely improve in function. But we wanted to focus on the natural history of functional improvement uninfluenced by these surgical procedures, so we excluded those who underwent a new total knee or hip replacement after the baseline assessment.

The MOST study protocol was approved by the institutional review boards at the University of Iowa, University of California San Francisco, University of Alabama, and Boston University Medical Center.

Outcome measures. We selected 3 definitions of MCII for WOMAC physical function. Our rationale for choosing these definitions was that they were anchored to patient-based indicators of improvement and defined meaningful improvement relative to baseline WOMAC physical function scores. All MCII definitions were dichotomous outcomes (improved/not improved) and were decreases in WOMAC physical function scores because lower scores on WOMAC represent less limitation. The first 2 definitions, MCII 26\% and MCII Tertile, were estimated from a study of 1362 people with knee pain reporting a "good, satisfactory effect with occasional episodes of pain or stiffness" following a 4-week course of nonsteroidal antiinflammatory drug (NSAID) ${ }^{9}$. The last definition, MCII 17\%, was from a study of 192 people with knee OA who underwent 3 to 4 weeks of inpatient rehabilitation ${ }^{10}$.

MCII 26\% and MCII 17\% defines meaningful improvement as a $26 \%$ and $17 \%$ decrease in WOMAC physical function (final value minus baseline value/baseline value), respectively, with a minimum absolute decrease of 2 out of 68. For instance, a baseline WOMAC physical function score of 30 would need a 5.1-point decrease to meet meaningful improvement for MCII $17 \%$ criteria, and a 7.8-point improvement to meet MCII 26\% criteria.

MCII Tertile defines meaningful improvement as absolute values (final value minus baseline value) dependent on baseline WOMAC physical function scores. We considered those with a decrease of 3.6, 8.0, and 13.9 out of 68 to reach meaningful improvement within low, medium, and high baseline tertile categories, respectively. These cutoff values came from the study of a 4-week course of NSAID in persons with knee pain ${ }^{9}$.

Baseline risk factors. All participants underwent bilateral weight-bearing posteroanterior (PA) and lateral fixed-flexion radiographic evaluation of the knee ${ }^{19}$. We noted the presence or absence of radiographic knee OA (ROA) in either knee. We defined ROA based on radiographic findings in either tibiofemoral or patellofemoral joints. For the tibiofemoral joint this was a Kellgren and Lawrence (KL) grade $>2$, and for the patellofemoral joint an osteophyte score $>2$, or any joint space narrowing score $>2$ with any osteophyte, sclerosis, or cyst score of $>1$ on a lateral plain-view radio$\operatorname{graph}^{20,21}$. The interrater reliability-weighted $\kappa$ for the KL grade at baseline was 0.80 . For persons with ROA, we noted whether subjects had ROA in 1 or both knees.

Subjects self-reported age in years, sex, and educational attainment as attending some college or not. Comorbidities were estimated as none or 1 or more with a validated self-report measure, the modified Charlson comorbidity index ${ }^{22}$. BMI was classified according to the World Health Organization categories ${ }^{23}$ and computed from standardized weight and height assessments. Knee pain (by visual analog scale) in the more painful knee was used for analysis and was quantified as the average knee pain over the past 30 days as measured on a horizontal line with 0 and 10 as endpoints. Walking speed was measured continuously in meters/second $(\mathrm{m} / \mathrm{s})$ from walking at a usual pace over 20 meters. Knee strength was classified in tertiles using the weaker knee as a data point, and was calculated from the mean of 4 isokinetic knee extensor torque repetitions at $60 \%$ measured in Newton-meters. Depressive symptoms were classified by risk of significant depressive symptomatology measured with the Center for Epidemiologic Studies Depression Scale (CES-D: 0-60) $\geq 16^{24}$. Physical activity was measured in tertiles with the Physical Activity Scale for the Elderly (PASE: 0-360) ${ }^{25}$.

We also examined whether subjects were or were not taking medications or had a steroid injection up to the baseline assessment. Specifically, we asked subjects if they took the following medications for arthritis every day or almost every day: aspirin, ibuprofen, acetaminophen, COX-2 inhibitors, or other NSAID. We also asked whether subjects had a steroid injection, such as cortisone, in either knee in the past 12 months from the baseline assessment.

Statistical analysis. To examine differences between people with and without clinically meaningful improvement, means and 95\% CI were applied for continuous variables and odds ratios and chi-squared tests for categorical variables. We used multiple logistic regression for each of the 3 definitions of MCII, mutually adjusting for all baseline risk factors. We applied the Hosmer-Lemeshow goodness-of-fit statistics to examine the regression models.

We investigated the association between the following baseline risk factors with clinically meaningful improvement based on existing evidence linking them to changes in function 1,2,19,26-28: age, sex, educational attain- 
ment, ROA, comorbidities, BMI, knee pain, walking speed, knee extensor strength, depressive symptoms, physical activity, and medications. We performed additional analyses restricted to those with ROA at baseline, given that these subjects may have a different frequency of improvement and associated risk factors from those without ROA. We examined the same baseline risk factors with the addition of the presence of ROA in 1 or both knees.

\section{RESULTS}

Of the 3026 subjects from the MOST study at baseline, 782 had WOMAC physical function scores less than 4, and 20 did not have complete data. At the 30-month followup, 187 had a new total hip or knee replacement, 31 were lost to followup, and 205 did not have complete data or did not complete the 30-month assessment, leaving 1801 subjects for analysis (Figure 1). Compared to those included for analysis, the excluded sample $(n=1225)$ had a lower percentage of women ( $56 \%$ vs $63 \%$ ), fewer people with ROA (52\% vs $59 \%$ ), and fewer people with comorbidities (40\% vs $45 \%$; all $\mathrm{p}<0.05)$. There was no difference in age, education, or BMI. The most frequently missing risk factor among the 1801 included in the analyses was ROA status $(n=15)$ followed by level of education $(n=13)$.

Of the included subjects, the mean age was 62.7 years (SD 8.0). Most were women (63\%), had some college education $(71 \%)$, had ROA (59\%), had no comorbidities $(55 \%)$, and were overweight $\left(\mathrm{BMI}=30.8, \mathrm{SD} 6.0 \mathrm{~kg} / \mathrm{m}^{2}\right)$. A little over one-third reported taking medication for arthritis or having a steroid injection in either knee at baseline (39\%). Most subjects had mild to moderate limitations in function (mean WOMAC 18.7, SD 11.2). Subject characteristics and baseline data are listed in Table 1. Using different definitions of MCII, clinically meaningful improvement occurred in 615 (34\%) for the MCII 26\% method, 704 (39\%) for the MCII 17\% method, and 425 (24\%) for the MCII Tertile method. There were 425 subjects meeting criteria for all 3 definitions of MCII. The percentage of subjects meeting
Table 1. Subject characteristics and modifiable factors at baseline $(\mathrm{n}=$ 1801).

Characteristic

Age, yrs (SD)

$62.7(8.0)$

Women, n (\%)

$1135(63)$

Some college, n $(\%)$

ROA, n (\%)

Patellofemoral ROA, n (\%)

Tibiofemoral ROA, n (\%)

Patellofemoral and tibiofemoral ROA, n (\%)

Comorbidities, n (\%) none

$\mathrm{BMI}, \mathrm{kg} / \mathrm{m}^{2}$ (SD)

Knee pain, VAS 0-10 (SD)

Walking speed, $\mathrm{m} / \mathrm{s}$ (SD)*

Knee strength, Newton meters (SD)**

Depressive symptoms, CES-D 0-60 (SD)

Physical activity, PASE 0-360 (SD)

$1279(71)$

1045 (58.5)

$80(4.5)$

604 (33.8)

$361(20.2)$

991 (55)

30.9 (6.0)

$3.0(2.2)$

$1.18(0.2)$

68 (36)

$8(7.7)$

171 (87)

Arthritis medications or steroid injection, n (\%)*** 698 (39)

Baseline WOMAC physical function score, 4-68 (SD) 18.7 (11.2)

* Walking speed measured in meters per second over a 20 -meter walk at a usual pace. ** Isokinetic knee extensor strength. Weaker value of 2 knees used for analysis. *** Arthritis medication taken every day or almost every day including aspirin, ibuprofen, acetaminophen, COX-2 inhibitors, or other nonsteroidal or antiinflammatory medications, or a steroid injection, such as cortisone, in either knee in the past 12 months. ROA: radiographic knee osteoarthritis; BMI: body mass index; VAS: visual analog scale; CES-D: Center for Epidemiologic Studies Depression Scale; PASE: Physical Activity Scale for the Elderly; WOMAC: Western Ontario McMaster Universities Osteoarthritis Index.

MCII across the range of baseline WOMAC physical function scores is depicted in Figure 2.

Baseline risk factors associated with meaningful improvement. For risk factors at baseline measured continuously, people with clinically important improvement had statistically significant lower BMI, faster walking speeds, and fewer depressive symptoms across all 3 MCII methods, less knee pain for the MCII 26\% and MCII Tertile methods, and

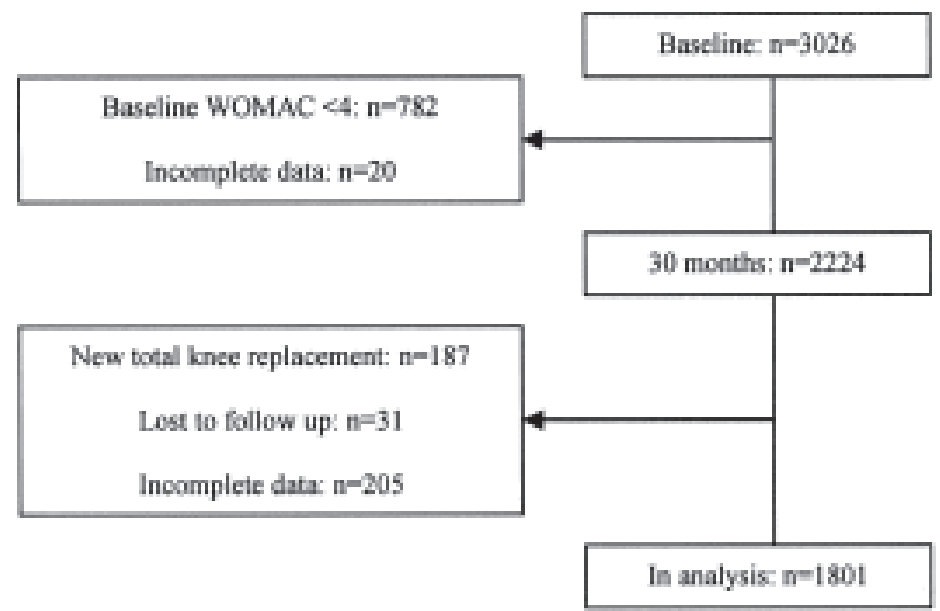

Figure 1. The progress of subjects from baseline to 30 months. WOMAC: Western Ontario McMaster Universities Osteoarthritis Index. 


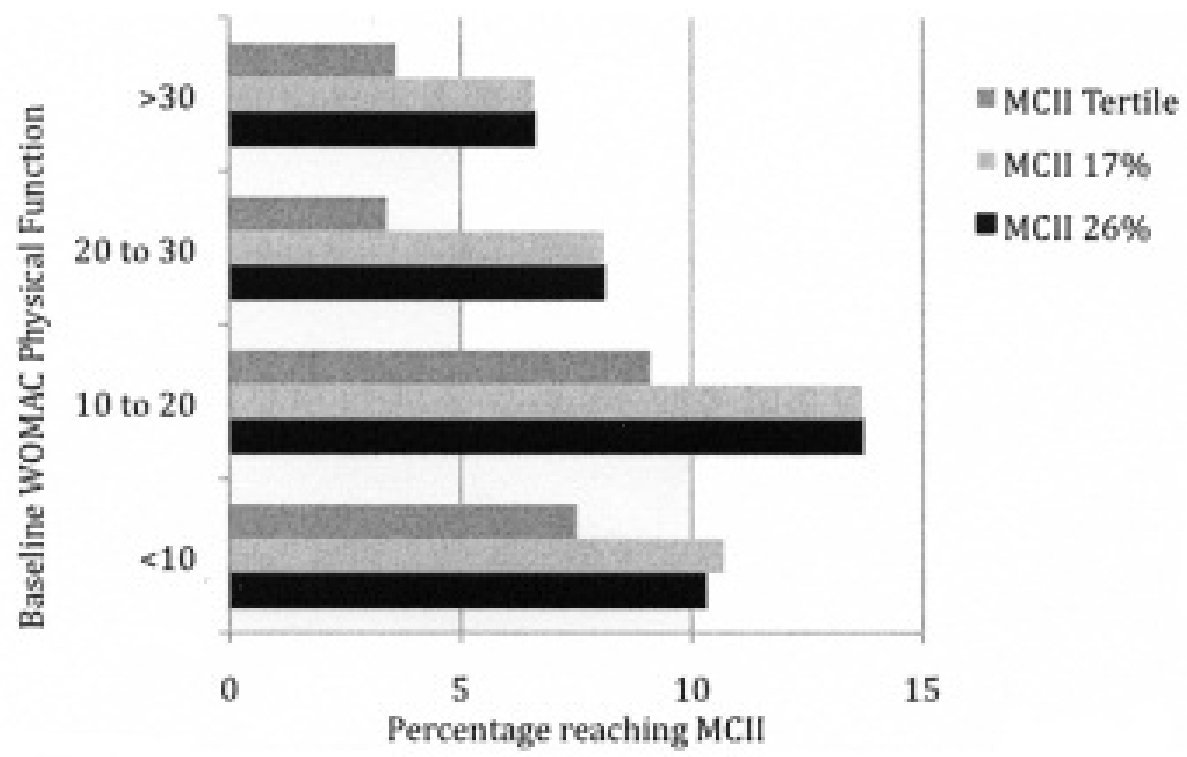

Figure 2. Percentages of subjects with meaningful improvement by minimal clinically important improvement (MCII) 26\%, MCII 17\%, and MCII Tertile by baseline WOMAC physical function score groups. MCII percentages refer to decrease in WOMAC physical function to reach meaningful clinically important improvement. Tertile refers to 3.6, 8.0, and 13.9 out of 68 for those in low, medium, and high baseline WOMAC physical function tertiles, respectively. WOMAC: Western Ontario McMaster Universities Osteoarthritis Index.

more knee strength using the MCII Tertile method, compared to their counterparts (Table 2). For risk factors measured categorically, people with ROA were $40 \%$ to $60 \%$ less likely to have clinically important improvement across all 3
MCII methods, and people with at least some college education were 1.3 times more likely to have clinically important improvement for the MCII 26\% and Tertile methods compared to their counterparts. People who take arthritis

Table 2. Differences (without MCII and with MCII) and 95\% CI in risk factors measured continuously at baseline between those with and without minimal clinically important improvement (MCII). All improvements indicated are mean (SD). Data in bold type represent $\mathrm{p}<0.05$.

\begin{tabular}{|c|c|c|c|c|c|c|c|c|c|}
\hline & \multicolumn{9}{|c|}{$\begin{array}{l}\text { MCII Definitions } \\
\text { MCII } 17 \% * *\end{array}$} \\
\hline & $\begin{array}{c}\text { Minimal } \\
\text { Improvement }\end{array}$ & $\begin{array}{c}\text { No Minimal } \\
\text { Improvement }\end{array}$ & $\begin{array}{l}\text { Difference } \\
(95 \% \mathrm{CI})\end{array}$ & $\begin{array}{c}\text { Minimal } \\
\text { improvement }\end{array}$ & $\begin{array}{l}\text { No Minimal } \\
\text { Improvement }\end{array}$ & $\begin{array}{l}\text { Difference } \\
(95 \% \mathrm{CI})\end{array}$ & $\begin{array}{c}\text { Minimal } \\
\text { Improvement }\end{array}$ & $\begin{array}{l}\text { No Minimal } \\
\text { Improvement }\end{array}$ & $\begin{array}{l}\text { Difference } \\
\text { t }(95 \% \mathrm{CI})\end{array}$ \\
\hline Age, yrs & $62.17(8)$ & $62.7(8)$ & $\begin{array}{c}0.0 \\
(-0.8,0.8)\end{array}$ & $62.7(8.0)$ & $62.7(8.0)$ & $\begin{array}{c}0.0 \\
(-0.7,0.8)\end{array}$ & $62.5(7.6)$ & $62.8(8.1)$ & $\begin{array}{c}0.3 \\
(-0.5,1.2)\end{array}$ \\
\hline BMI, $\mathrm{kg} / \mathrm{m}^{2}$ & $30.2(5.8)$ & $31.2(6.0)$ & $\begin{array}{c}1.0 \\
(0.4,1.6)\end{array}$ & $30.3(5.8)$ & $31.2(6.1)$ & $\begin{array}{c}0.9 \\
(0.3,1.5)\end{array}$ & $30.0(5.6)$ & $31.1(6.1)$ & $\begin{array}{c}1.1 \\
(0.5,1.8)\end{array}$ \\
\hline Walk speed, $\mathrm{m} / \mathrm{s}$ & $1.21(0.2)$ & $1.17(0.2)$ & $\begin{array}{c}-0.04 \\
(-0.06,-0.02)\end{array}$ & $1.20(0.2)$ & $1.17(0.2)$ & $\begin{array}{c}-0.03 \\
(-0.06,-0.02)\end{array}$ & $1.22(0.2)$ & $1.17(0.2)$ & $\begin{array}{c}-0.05 \\
(-0.07,-0.03)\end{array}$ \\
\hline $\begin{array}{l}\text { Knee strength, } \\
\text { Newton-meters }\end{array}$ & $70.7(36.1)$ & $67.1(35.8)$ & $\begin{array}{c}-3.6 \\
(-7.3,0.0)\end{array}$ & $70.1(36.3)$ & $67.1(35.8)$ & $\begin{array}{c}-2.9 \\
(-6.5,0.6)\end{array}$ & $73.0(35.5)$ & 66.9 (36.1) & $\begin{array}{c}-6.1 \\
(-10.3,-2.1)\end{array}$ \\
\hline $\begin{array}{l}\text { Depressive } \\
\text { symptoms } \\
\text { (CES-D 0-60) }\end{array}$ & $7.3(7.3)$ & $8.5(8.0)$ & $\begin{array}{c}1.2 \\
(0.5,2.0)\end{array}$ & $7.4(7.3)$ & $8.5(8.0)$ & $\begin{array}{c}1.1 \\
(0.4,1.8)\end{array}$ & $7.4(7.4)$ & $8.3(7.9)$ & $\begin{array}{c}0.9 \\
(0.1,1.7)\end{array}$ \\
\hline
\end{tabular}

$* 26 \%$ decrease in WOMAC physical function to reach MCII. ** $17 \%$ decrease in WOMAC physical function to reach minimal clinically important improvement. *** 3.6, 8.0, and 13.9 out of 68 for those in low, medium, and high baseline WOMAC physical function tertiles, respectively. WOMAC: Western Ontario McMaster Universities Osteoarthritis Index; BMI: body mass index; VAS: visual analog scale; CES-D: Center for Epidemiologic Studies Depression Scale; PASE: Physical Activity Scale for the Elderly. 
medication or who had had a steroid injection in the last 12 months were $20 \%$ to $40 \%$ less likely to have clinically important improvement across all 3 MCII methods compared with their counterparts (Table 3).

After mutual adjustment for all risk factors, ROA status and walking speed remained associated with MCII across all 3 methods of estimating MCII. People with ROA were $40 \%$ to $50 \%$ less likely to have clinically important improvement compared to those without ROA, and people able to walk 1.0 $\mathrm{m} / \mathrm{s}$ faster than their counterparts were 1.9 to 2.0 times more likely to have clinically important improvement (Table 4).

Analysis including only those with ROA. Of the 1045 subjects with ROA, 470 (45\%) had ROA in 1 knee and 575 $(55 \%)$ had ROA in both knees. Clinically meaningful improvement occurred in 288 (28\%) for the MCII $26 \%$ method, $346(33 \%)$ for the MCII 17\% method, and 179

Table 3. Odds of minimal clinical important improvement (MCII) and 95\% CI for risk factors measured categorically at baseline. Higher odds represent higher likelihood of meaningful improvement. Data in bold type represent confidence intervals that do not cross 1.0.

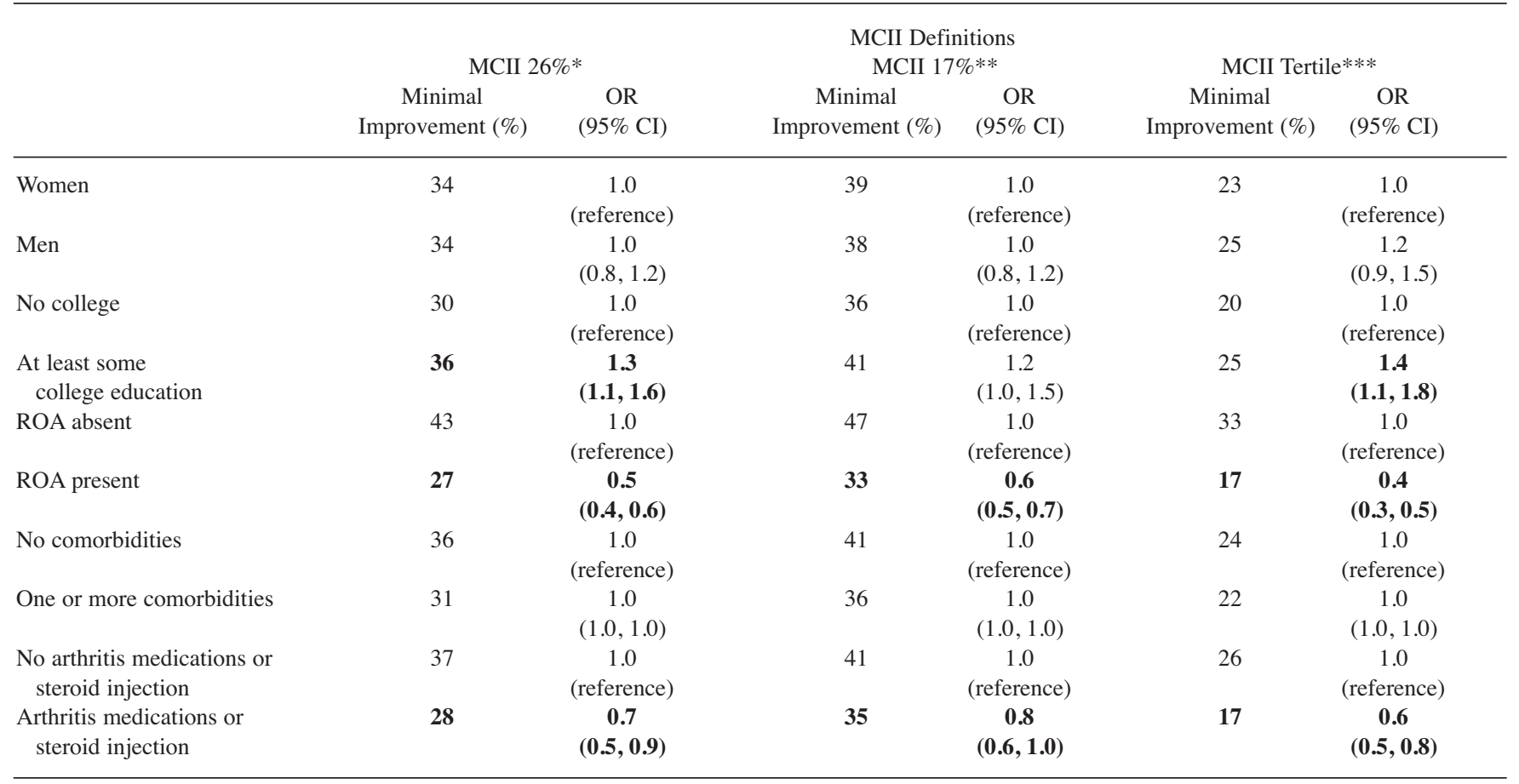

* $26 \%$ decrease in WOMAC physical function to reach meaningful clinically important improvement. ** $17 \%$ decrease in WOMAC physical function to reach meaningful clinically important improvement. ***3.6, 8.0, and 13.9 out of 68 for those in low, medium, and high baseline WOMAC physical function tertiles, respectively. ROA: radiographic knee osteoarthritis; WOMAC: Western Ontario McMaster Universities Osteoarthritis Index.

Table 4. OR and 95\% CI among all subjects for risk factors associated with minimal clinically important improvement (MCII) in WOMAC physical function mutually adjusted for other factors*; with higher OR representing greater likelihood of MCII.

\begin{tabular}{|c|c|c|c|c|c|}
\hline & & $\mathrm{n}$ & MCII $26 \% * *$ & $\begin{array}{l}\text { MCII Definitions } \\
\quad \text { MCII } 17 \%^{\dagger}\end{array}$ & $\begin{array}{c}\text { MCII } \\
\text { Tertile }^{\dagger \dagger}\end{array}$ \\
\hline \multirow[t]{2}{*}{ ROA } & Absent & 756 & 1.0 (reference) & 1.0 (reference) & 1.0 (reference) \\
\hline & Present & 1045 & $0.6(0.4,0.7)$ & $0.6(0.5,0.7)$ & $0.5(0.4,0.6)$ \\
\hline $\begin{array}{l}\text { Walking speed, } \\
\mathrm{m} / \mathrm{s}\end{array}$ & $\begin{array}{l}\text { OR of MCII } \\
\text { per } 1.0 \mathrm{~m} / \mathrm{s}\end{array}$ & 1801 & $1.9(1.0,3.4)$ & $1.9(1.1,3.3)$ & $2.0(1.0,3.9)$ \\
\hline \multicolumn{3}{|c|}{ No. of subjects included in logistic models } & 1768 & 1768 & 1768 \\
\hline \multicolumn{3}{|c|}{$\mathrm{p}$, Hosmer-Lemeshow test, 8 degrees of freedom } & 0.08 & 0.61 & 0.32 \\
\hline
\end{tabular}

* Mutually adjusted for age, gender, education, comorbidities, body mass index, knee pain, knee strength, depressive symptoms, physical activity, and medication usage at baseline. $* * 26 \%$ decrease in WOMAC physical function to reach minimal clinically important improvement. ${ }^{\dagger} 17 \%$ decrease in WOMAC physical function to reach minimal clinically important improvement. ${ }^{\dagger \dagger} 3.6,8.0$, and 13.9 out of 68 for those in low, medium, and high baseline WOMAC physical function tertiles, respectively. ROA: radiographic knee osteoarthritis; WOMAC: Western Ontario McMaster Universities Osteoarthritis Index. 
(17\%) for the MCII Tertile method. People with clinically important improvement had faster walking speeds and were more likely to have ROA in 1 knee across all 3 methods of estimating MCII, fewer depressive symptoms for the MCII $26 \%$ and MCII $17 \%$ methods, and less knee pain and a lower BMI for the MCII 26\% and MCII 17\% methods, respectively, compared to their counterparts (data not shown). After mutual adjustment, we found persons in the highest strength tertile to be 1.9 to 2.2 times more likely to have clinically meaningful improvement across all 3 methods of estimating MCII compared with those in the lowest strength tertile (Table 5).

\section{DISCUSSION}

A substantial percentage of subjects $(24 \%-39 \%)$ in our study had clinically important improvements in WOMAC physical function 30 months after initial assessment. People who had clinically important improvement had a lower BMI, faster walking speeds, and fewer depressive symptoms across all 3 definitions of MCII unadjusted for other risk factors. After mutual adjustment for other risk factors, people who improved walked faster and did not have radiographic evidence of knee OA at baseline compared with those who did not improve.

The MCII allows one to estimate how many people had clinically meaningful improvement, and represents the smallest improvement in score that can be regarded as important. Limiting investigation of longitudinal changes to group-level analysis, such as mean change and SD summary statistics, may create a perception that subjects' functional status is fixed. For instance, Botha-Scheepers and colleagues recently reported little change in functional limitations in a cohort of 115 people with symptomatic knee and hip OA over 2 years, as evidenced by a mean increase of 2.2 (SD 12.7) in WOMAC physical function ${ }^{29}$. We found simi- lar mean change in WOMAC physical function (mean 0.7, SD 9.8), but our evaluation of change at the level of the individual revealed a large percentage of people with substantial improvement. Improvements in functional limitation have been reported. Most recently, Ayis and Dieppe found that $107(19.6 \%)$ of 545 subjects with functional limitation at baseline had improvement when measured 8 years later, although these changes were not necessarily measured at a level of clinically meaningful improvement ${ }^{14}$.

Our study findings reveal that people with ROA had at least a $40 \%$ reduction in odds of clinically important improvement in function across all 3 definitions of MCII, compared with those without ROA. Several studies support the notion that ROA influences changes in function. Roos and colleagues found that the presence of tibiofemoral OA was predictive of decline in sport and recreation activities 4 to 10 years later ${ }^{30}$, and Davis and coauthors reported people with ROA at baseline were more likely to report difficulty with mobility-related activities 10 years later than those without ROA ${ }^{31}$. While some studies did not find an association between ROA status and function ${ }^{32,33}$, several possibilities exist for this association in our study. First, we had ample power and heterogeneity of age to detect this association. We included 1801 people who were at least 50 years of age. Second, our primary outcome was clinically meaningful improvement in function, which was not used in previous studies ${ }^{32,33}$. Lastly, our study took knee radiographs with a standing fixed-flexion body position that has been shown to have high test-retest reliability ${ }^{34}$. Other studies used a full-extended position of the knee ${ }^{32,33}$, which has been shown to be less reliable and accurate with estimating the severity of radiographic changes in the knee than a standing fixed-flexion body position ${ }^{35}$.

Walking speed over 20 meters was also found to be associated with meaningful improvement in function across all 3

Table 5. OR and 95\% CI among persons with ROA at baseline for risk factors associated with minimal clinically important improvement (MCII) in WOMAC physical function mutually adjusted for other factors*; higher OR representing greater likelihood of MCII.

\begin{tabular}{lccccc}
\hline \multicolumn{1}{c}{ Mean (SD) } & $\mathrm{n}$ & MCII 26\%** & $\begin{array}{c}\text { MCII Definitions } \\
\text { MCII 17\% }\end{array}$ & $\begin{array}{c}\text { MCII } \\
\text { Tertile }^{\dagger \dagger}\end{array}$ \\
\hline Knee strength, Newton meters & & & & \\
Low & $27.1(9.7)$ & 316 & 1.0 (reference) & 1.0 (reference) & 1.0 (reference) \\
Medium & $54.75(8.1)$ & 313 & $1.5(1.0,2.2)$ & $1.4(1.0,2.0)$ & $1.6(1.0,2.6)$ \\
High & $104.0(28.3)$ & 315 & $1.9(1.1,3.0)$ & $1.7(1.1,2.7)$ & $2.2(1.2,4.0)$ \\
No. of subjects included in logistic models & 935 & 935 & 935 \\
p. Hosmer-Lemeshow test, 8 degrees of freedom & 0.18 & 0.31 & 0.37 \\
\hline
\end{tabular}

\footnotetext{
* Mutually adjusted for age, gender, education, comorbidities, body mass index, knee pain, depressive symptoms, physical activity, ROA in 1 or both knees, and medication usage at baseline. $* * 26 \%$ decrease in WOMAC physical function to reach minimal clinically important improvement. ${ }^{\dagger} 17 \%$ decrease in WOMAC physical function to reach minimal clinically important improvement. ${ }^{\dagger} 3.6,8.0$, and 13.9 out of 68 for those in low, medium, and high baseline WOMAC physical function tertiles, respectively. WOMAC: Western Ontario McMaster Universities Osteoarthritis Index; ROA: radiographic knee osteoarthritis.
} 
definitions of MCII. This is consistent with other studies, which show that slow walking speed in older adults is associated with a variety of adverse outcomes including incident functional limitation ${ }^{18}$, hospital admission ${ }^{36}$, and mortality ${ }^{37-39}$. Our findings extend walking speed as a marker of meaningful improvement in younger adults over the age of 50 years with or at high risk of knee OA. The speed of walking can be considered an estimate of walking ability. Given that the WOMAC physical function subscale measures self-reported difficulty with walking and several tasks for which walking is prerequisite, we expected faster walking speeds to be associated with clinically important improvement in function.

Certainly it is plausible that interventions that took place over 30 months may be responsible for subsequent meaningful improvements in function. Our cohort had mild to moderate limitations in function at baseline, as evidenced by a mean WOMAC physical function score of 18.7. Hence most study subjects would not have been referred for physical rehabilitation. We found persons taking prescription medication or those who had a steroid injection by the baseline examination to be less likely to have meaningful improvements in function in the unadjusted analysis. It is likely that these individuals had greater functional involvement and were thus less likely to improve. The association of persons starting to take medications over the 30-month period with meaningful improvement in function would be confounded by indication ${ }^{40}$.

There are some limitations in our study. First, we employed cutoff values for MCII from studies that used patient-anchored definitions of meaningful improvements, and not other anchoring methods such as clinician or consensus cutoffs. Second, Gill and colleagues have recently suggested that fluctuations between states of ability and inability are much higher when outcomes are measured monthly compared to longer assessment intervals ${ }^{41}$. Since we calculated change in WOMAC physical function using only 2 reference points, baseline and 30 months, it is possible that the proportion of those with transient meaningful improvement on a monthly basis may be even more common than we estimated over 30 months. Future studies should employ repeated measures within shorter time intervals to investigate the cumulative frequency of meaningful improvement and time course of fluctuations in function. Third, we measured function using a self-report instrument, and lower rates of improvement have been reported for performance-based measures compared to self-report measures $^{33}$. Future study should incorporate both self-report and performance-based outcomes to measure function better. Fourth, we used only 1 measure, the visual analog scale, to estimate knee pain, a method that may underestimate the ability of knee pain to predict meaningful improvements in function. We were reluctant to use the WOMAC pain score as a modifiable factor because of its high correlation with the WOMAC physical function score ${ }^{42}$. Fifth, potential bias may exist in our estimate of $24 \%-39 \%$ of subjects achieving MCII. We excluded those with new total joint replacements, and included those who had or were at high risk of symptomatic knee OA. Also, it is important to note that the percentage of those with meaningful improvement will naturally be higher using the MCII 17\% cutpoint compared with MCII 26\%, given that less change is needed for meaningful improvement. Sixth, we arbitrarily selected a WOMAC physical function cutoff of $4 / 68$ to represent those with at least a minimal amount of functional limitation. We have analyzed the data using other cutoffs (range 3-6) and found similar percentages of recovery across all methods of calculating MCII. Lastly, we did not differentiate between persons who had 1 versus 2 painful knees, which could have an effect on meaningful improvement in function. Future research should investigate whether persons with 1 painful knee are more likely to have meaningful improvement in function compared with those with 2 painful knees.

Meaningful improvement is common among those with generally mild to moderate self-reported limitations in function who have or are at high risk for knee OA over a 30 -month period. Our study found a robust percentage of people to have these improvements irrespective of the method used to estimate improvement. We emphasize that our definition of meaningful improvement excluded those with unchanged or worsening WOMAC physical function scores over 30 months. People without radiographic evidence of knee OA and those with fast walking speeds are more likely to have improvements than those with ROA and slower walking speeds. Healthcare providers may want to consider these risk factors when determining who may benefit from therapeutic intervention.

\section{ACKNOWLEDGMENT}

We acknowledge the MOST study subjects for their participation.

\section{REFERENCES}

1. Guccione AA, Felson DT, Anderson JJ. Defining arthritis and measuring functional status in elders: methodological issues in the study of disease and physical disability. Am J Public Health 1990;80:945-9.

2. Sharma L, Cahue S, Song J, Hayes K, Pai YC, Dunlop D. Physical functioning over three years in knee osteoarthritis: role of psychosocial, local mechanical, and neuromuscular factors. Arthritis Rheum 2003;48:3359-70.

3. Belo JN, Berger MY, Koes BW, Bierma-Zeinstra SM. The prognostic value of the clinical ACR classification criteria of knee osteoarthritis for persisting knee complaints and increase of disability in general practice. Osteoarthritis Cartilage 2009; $17: 1288-92$

4. van der Waal JM, Bot SD, Terwee CB, van der Windt DA, Scholten RJ, Bouter LM, et al. Course and prognosis of knee complaints in general practice. Arthritis Rheum 2005;53:920-30.

5. Ayis S, Ebrahim S, Williams S, Juni P, Dieppe P. Determinants of reduced walking speed in people with musculoskeletal pain. J Rheumatol 2007;34:1905-12. 
6. Dieppe P, Cushnaghan J, Tucker M, Browning S, Shepstone L. The Bristol 'OA500 study': progression and impact of the disease after 8 years. Osteoarthritis Cartilage 2000;8:63-8.

7. Peters TJ, Sanders C, Dieppe P, Donovan J. Factors associated with change in pain and disability over time: a community-based prospective observational study of hip and knee osteoarthritis. $\mathrm{Br}$ J Gen Pract 2005;55:205-11.

8. Beaton DE, Bombardier C, Katz JN, Wright JG. A taxonomy for responsiveness. J Clin Epidemiol 2001;54:1204-17.

9. Tubach F, Ravaud P, Baron G, Falissard B, Logeart I, Bellamy N, et al. Evaluation of clinically relevant changes in patient reported outcomes in knee and hip osteoarthritis: the minimal clinically important improvement. Ann Rheum Dis 2005;64:29-33.

10. Angst F, Aeschlimann A, Michel BA, Stucki G. Minimal clinically important rehabilitation effects in patients with osteoarthritis of the lower extremities. J Rheumatol 2002;29:131-8.

11. Johnson SR, Archibald A, Davis AM, Badley E, Wright JG, Hawker GA. Is self-reported improvement in osteoarthritis pain and disability reflected in objective measures? J Rheumatol 2007:34:159-64.

12. Mallen CD, Peat G, Thomas E, Lacey R, Croft P. Predicting poor functional outcome in community-dwelling older adults with knee pain: prognostic value of generic indicators. Ann Rheum Dis 2007;66:1456-61

13. Thomas E, Peat G, Mallen C, Wood L, Lacey R, Duncan R, et al. Predicting the course of functional limitation among older adults with knee pain: do local signs, symptoms and radiographs add anything to general indicators? Ann Rheum Dis 2008;67:1390-8.

14. Ayis S, Dieppe P. The natural history of disability and its determinants in adults with lower limb musculoskeletal pain. J Rheumatol 2009;36:583-91.

15. Rejeski WJ, Ip EH, Marsh AP, Zhang Q, Miller ME. Obesity influences transitional states of disability in older adults with knee pain. Arch Phys Med Rehabil 2008;89:2102-7.

16. Jinks C, Jordan K, Croft P. Osteoarthritis as a public health problem: the impact of developing knee pain on physical function in adults living in the community: (KNEST 3). Rheumatology 2007;46:877-81.

17. Cesari M, Kritchevsky SB, Penninx BW, Nicklas BJ, Simonsick EM, Newman AB, et al. Prognostic value of usual gait speed in well-functioning older people - results from the Health, Aging and Body Composition Study. J Am Geriatr Soc 2005;53:1675-80.

18. Guralnik JM, Ferrucci L, Pieper CF, Leveille SG, Markides KS, Ostir GV, et al. Lower extremity function and subsequent disability: consistency across studies, predictive models, and value of gait speed alone compared with the short physical performance battery. J Gerontol A Biol Sci Med Sci 2000;55:M221-31.

19. Felson DT, Niu J, Guermazi A, Roemer F, Aliabadi P, Clancy M, et al. Correlation of the development of knee pain with enlarging bone marrow lesions on magnetic resonance imaging. Arthritis Rheum 2007;56:2986-92.

20. Kellgren JH, Lawrence JS. Radiological assessment of osteo-arthrosis. Ann Rheum Dis 1957;16:494-502.

21. Felson DT, McAlindon TE, Anderson JJ, Naimark A, Weissman $\mathrm{BW}$, Aliabadi P, et al. Defining radiographic osteoarthritis for the whole knee. Osteoarthritis Cartilage 1997;5:241-50.

22. Katz JN, Chang LC, Sangha O, Fossel AH, Bates DW. Can comorbidity be measured by questionnaire rather than medical record review? Med Care 1996;34:73-84.

23. World Health Organization. Obesity: preventing and managing the global epidemic. Report of a WHO Consultation. WHO Technical Report Series 894. Geneva: World Health Organization; 2000.

24. Radloff L. The CES-D Scale: A self-report depression scale for research in the general population. Appl Psychol Meas 1977;1:385-401.
25. Washburn RA, Smith KW, Jette AM, Janney CA. The Physical Activity Scale for the Elderly (PASE): development and evaluation. J Clin Epidemiol 1993;46:153-62.

26. Jordan J, Luta G, Renner J, Dragomir A, Hochberg M, Fryer $\mathrm{J}$. Knee pain and knee osteoarthritis severity in self-reported task specific disability: the Johnston County Osteoarthritis Project. J Rheumatol 1997;24:1344-9.

27. Eyler AA, Brownson RC, Bacak SJ, Housemann RA. The epidemiology of walking for physical activity in the United States. Med Sci Sports Exerc 2003;35:1529-36.

28. Penninx BW, Guralnik JM, Ferrucci L, Simonsick EM, Deeg DJ, Wallace RB. Depressive symptoms and physical decline in community-dwelling older persons. JAMA 1998;279:1720-6.

29. Botha-Scheepers S, Watt I, Rosendaal FR, Breedveld FC, Hellio le Graverand MP, Kloppenburg M. Changes in outcome measures for impairment, activity limitation, and participation restriction over two years in osteoarthritis of the lower extremities. Arthritis Rheum 2008;59:1750-5.

30. Roos EM, Bremander AB, Englund M, Lohmander LS. Change in self-reported outcomes and objective physical function over 7 years in middle-aged subjects with or at high risk of knee osteoarthritis. Ann Rheum Dis 2008;67:505-10.

31. Davis MA, Ettinger WH, Neuhaus JM, Mallon KP. Knee osteoarthritis and physical functioning: evidence from the NHANES I Epidemiologic Followup Study. J Rheumatol 1991;18:591-8.

32. Barker K, Lamb SE, Toye F, Jackson S, Barrington S. Association between radiographic joint space narrowing, function, pain and muscle power in severe osteoarthritis of the knee. Clin Rehabil 2004; 18:793-800.

33. Miller ME, Rejeski WJ, Messier SP, Loeser RF. Modifiers of change in physical functioning in older adults with knee pain: the Observational Arthritis Study in Seniors (OASIS). Arthritis Rheum 2001;45:331-9.

34. Kothari M, Guermazi A, von Ingersleben G, Miaux Y, Sieffert M, Block JE, et al. Fixed-flexion radiography of the knee provides reproducible joint space width measurements in osteoarthritis. Eur Radiol 2004;14:1568-73.

35. Buckland-Wright JC, Macfarlane DG, Williams SA, Ward RJ. Accuracy and precision of joint space width measurements in standard and macroradiographs of osteoarthritic knees. Ann Rheum Dis 1995;54:872-80.

36. Studenski S, Perera S, Wallace D, Chandler JM, Duncan PW, Rooney E, et al. Physical performance measures in the clinical setting. J Am Geriatr Soc 2003;51:314-22.

37. Perera S, Studenski S, Chandler JM, Guralnik JM. Magnitude and patterns of decline in health and function in 1 year affect subsequent 5-year survival. J Gerontol 2005;60:894-900.

38. Hardy SE, Perera S, Roumani YF, Chandler JM, Studenski SA. Improvement in usual gait speed predicts better survival in older adults. J Am Geriatr Soc 2007;55:1727-34.

39. Dumurgier J, Elbaz A, Ducimetiere P, Tavernier B, Alperovitch A, Tzourio C. Slow walking speed and cardiovascular death in well functioning older adults: prospective cohort study. BMJ 2009;339:b4460.

40. Signorello LB, McLaughlin JK, Lipworth L, Friis S, Sorensen HT, Blot WJ. Confounding by indication in epidemiologic studies of commonly used analgesics. Am J Ther 2002;9:199-205.

41. Gill TM, Hardy SE, Williams CS. Underestimation of disability in community-living older persons. J Am Geriatr Soc 2002;50:1492-7.

42. Terwee CB, van der Slikke RM, van Lummel RC, Benink RJ, Meijers WG, de Vet HC. Self-reported physical functioning was more influenced by pain than performance-based physical functioning in knee-osteoarthritis patients. J Clin Epidemiol 2006;59:724-31. 